Running head: ABSORPTION, MENTALIZING, AND MYSTICISM: SENSING THE PRESENCE OF THE DIVINE

\title{
Absorption, Mentalizing, and Mysticism: Sensing the Presence of the Divine
}

ACCEPTED FOR PUBLICATION IN THE JOURNAL FOR THE COGNITIVE SCIENCE OF

$R E L I G I O N$ [this is an unedited pre-print and differs slightly from final version]

Coleman, T. J., III, Bartlett, J. E., Holcombe, J. M., Swanson, S. B., Atkinson, A. R., Silver, C. F., \& Hood, R. W. Jr., (in press). Absorption, Mentalizing, and Mysticism: Sensing the presence of the divine. Journal for the Cognitive Science of Religion.

a, b Thomas J. Coleman III, a, c James E. Bartlett, d Jenny M. Holcombe, e Sally B.

Swanson, ${ }^{\mathrm{f}}$ Andrew Atkinson, ${ }^{\mathrm{g}}$ Christopher F. Silver, and ${ }^{\mathrm{h}}$ Ralph W. Hood Jr.

${ }^{\text {a }}$ Coventry University, Centre for Advances in Behavioral Science; Brain, Belief, and

Behavior Research Lab

${ }^{\mathrm{b}}$ Grand Valley State University, Department of Psychology

${ }^{c}$ Arden University, School of Psychology, Law, and Social Science

${ }^{\mathrm{d}}$ University of Tennessee at Chattanooga, School of Nursing

${ }^{\mathrm{e}}$ University of Maine, Department of Psychology

${ }^{\mathrm{f}}$ Independent scholar

g University of Tennessee at Chattanooga, Learning and Leadership Doctoral

Program

${ }^{\mathrm{h}}$ University of Tennessee at Chattanooga, Department of Psychology

Corresponding author:

Thomas J. Coleman III

1703 Seagull Lane Hixson TN, 37343

Colema56@uni.coventry.ac.uk

ORCID ID: 0000-0002-3003-5090 


\begin{abstract}
Research suggests trait absorption, individual differences in Theory of Mind (ToM), and orthopraxical training are important for explaining a variety of extraordinary experiences typically associated with religion. However, no studies exist quantifying ToM ability or testing its relationship with trait absorption in the prediction of what is arguably the most ubiquitous type of extraordinary experience - the mystical experience. To address this, two exploratory studies were conducted using a sample of meditators $(N=269)$ and undergraduate students $(N=123)$. In study one, regression analyses revealed weekly religious/spiritual practice, absorption, and mentalizing predict increased mystical experiences. Moreover, moderation analysis indicated the absorptionmysticism relationship is stronger among individuals with lower mentalizing ability. Study two only replicated the relationship of absorption and weekly practice with mysticism. These studies highlight the robust contribution of absorption in mystical experiences and suggest a more dynamic role for mentalizing than is accounted for in the current literature.
\end{abstract}

Keywords: Mysticism, Absorption, Mentalizing, Religious Experience, Ritual 


\subsection{Introduction}

A recurring theme throughout religious traditions is the ability to dissociate from mundane reality and absorb into something greater, entering into trance-like, altered states of consciousness. Evidence for dissociative experiences are found throughout the deep history of human evolution (Rossano 2006), for example, in the phosphene-patterned imagery of some Upper Paleolithic art (Lewis-Williams 2010). Cognitive scientists of religion theorize that these extraordinary experiences are produced by ordinary species-typical human socio-cognitive capabilities, such as mentalizing, also known as theory of mind (ToM; Boyer and Bergstrom 2008; Greenway 2018; McCauley 2011). In support of this theorization, some research has identified a positive relationship between mentalizing and belief in God (Norenzayan et al. 2012) or prayer practice (Edman et al. 2018). Additionally, recent research suggests that ritual practices and a specific type of attentional processing captured by trait absorption may enable the cultivation of richer and more intense divine religious or spiritual experiences (Luhrmann 2012, 2013). Past research has identified a strong link between absorption and self-transcendent, mystical experiences (Granqvist et al. 2005; Ladilaw, Dwivedi, Naito and Gruzelier 2005; Spanos and Moretti 1988). However, no studies exist exploring the individual and combined effects of these cognitive variables (mentalizing, absorption) and ritual practice on self-reported mystical experiences.

Across two studies, the present research applies hierarchical regression analyses to explore the relationship of mentalizing, absorption, frequency and duration of ritual practice, on selfreported mystical experiences.

\subsection{Mysticism}


From William James (1902/1985) to the present day, some scholars have placed mystical, transcendent-like experiences at the core of religious traditions and beliefs (cf. Hood 2013; Hood, Hill and Spilka 2018). Wiebe (2013) argues that these experiences are integral to the evolution of religion because they provide experiential confirmation of unseen worlds in the minds of believers. It is difficult to understand many religious traditions in the absence of an experiential grounding, because their adherents believe they are responding to something-something that can be experienced (Hood 2001). Regardless, mysticism is not purely reducible to the category of religion because these experiences can occur in atheists and be framed in nonreligious ways (Coleman, Swhajor-Biesemann, Giamundo, Vance, Hood and Silver 2016; James 1902/1985).

Mysticism, as a social scientific category, has included phenomena ranging from experiences of "pure consciousness," to specific visual, auditory, and tactile hallucinations, and unusual religious or spiritual experiences more broadly (Andersen et al. 2014; Hood et al. 2018; Luhrmann 2013). Although there is no scholarly consensus on how to define or measure mysticism, the present study follows Stace's (1960) focus on a phenomenological "common core" to mysticism that includes intense experiences of timelessness, spacelessness, inner subjectivity, and noetic quality. ${ }^{1}$ Stace's definition emphasizes mysticism as, at one and the same time, a sensory based experience of unity (perceived as a union with some Absolute) and a unitive experience that is devoid of content and not sensory-based (Hood 2013).

Mystical experiences are reported across cultural and religious borders; however, Eastern contemplative traditions, such as the sample of meditators utilized in study one, have usually placed greater emphasis on their cultivation and desirability when compared to the Western

\footnotetext{
${ }^{1}$ For in-depth discussions of Stace's phenomenology see Klein, Silver, Streib, Hood, and Coleman (2016) and Hood (2001). For methodological discussions on the complexity of defining and measuring "mysticism," see Hood (2001, 2013), Andersen et al. (2014), and Katz (1978).
} 
world's fixation on doctrine and belief (Partridge and Gabriel 2003). Further, many traditional Eastern cultural teachings and practices explicitly aim to achieve the phenomenological states associated with mysticism (Dahl, Lutz and Davidson 2015). This is in contrast to the influence of Protestantism on Western culture, which emphasizes occasional spontaneity and sudden onset while deemphasizing their direct pursuit (Taves 1999). Regardless, in both Eastern and Western cultures self-reported features of mystical experiences include ineffability, ego-dissolution, loss of sense of time and space, a sense of something greater than oneself, and union with this greaterthan-self (Hood 2001, 2013; Stace 1960).

Mystical experiences can be elicited under certain conditions and settings, such as experiences in nature, religious settings (e.g., prayer, ritual), drugs, sexual intercourse, sensory deprivation, and even under "sham" treatment conditions (Andersen et al. 2014; Hood 1977, 2001). Although mystical experiences recruit numerous brain regions (van Elk and Aleman 2017), of interest to the current study is their overlap with areas involved in mentalizing and absorption-like states (Beauregard and Paquette 2006; Cristofori et al. 2016; Mitchell 2009; van Elk and Aleman 2017; Wickramasekera 2015). Moreover, studying the contributions of mentalizing, absorption, and ritual practice to mystical experiences supports recent calls for cognitive science of religion to focus less on belief and more on the role of experiences that support belief in relation to universal cognitive processes (Van Leeuwen and van Elk 2018; Wiebe 2013).

\subsection{Mentalizing}

Mentalizing is theorized as one of the key cognitive processes underlying religious belief and experience (Gervais 2013; McCauley 2011). As a construct, mentalizing arguably involves several distinct and context-dependent subprocesses - a discussion of which is beyond the scope of the current paper (for reviews, see Lindeman and Lipsanen 2017; Mitchell 2009). However, in the 
broadest sense, theorists agree that mentalizing is the ability to reason about the mental states of others and one's own mental states (Gervais 2013; Greenway 2018; Mitchell 2009; Reddish, Tok, and Kundt 2016). The centrality of mentalizing to the psychological and cognitive study of religion has longstanding theoretical importance (Boyer 1992), which has only recently undergone empirical exploration. Because believing in and communicating with supernatural agents is psychologically similar to believing in and communicating with ordinary human minds, mentalizing ability may predispose an individual to or facilitate the transmission of supernatural beliefs (Barrett 2004; Gervais 2013; Schjoedt et al. 2009).

Research suggests that mentalizing ability can constrain and facilitate belief in supernatural agents in general (Norenzayan et al. 2012; Rosenkranz and Charlton 2013) and the character of God concepts (Schaap-Jonker et al. 2013) or specific religious orientation (Caldwell-Harris et al. 2011; Brezis 2012; Visuri 2012). More generally, difficulties in mentalizing ability associated with the autism spectrum continuum are thought to be related to lower levels of religiosity (Norenzayan et al. 2012), whereas hyper-mentalizing abilities associated with psychotic-spectrum conditions, such as schizophrenia, relate to higher religiosity and/or paranormal beliefs (Willard and Norenzayan 2017; Wlodarski and Pearce 2016). However, several studies have failed to identify a significant relationship between mentalizing and religious belief, or found only a weak relationship (e.g., Coleman 2016; Jack et al. 2016; Maij et al. 2017; Reddish et al. 2016). Nevertheless, mentalizing has been consistently related to reported experiences of the divine, such as prayer or God image, which is the experienced relationship between an individual and deity (Edman et al. 2018; Greenway 2018; Jack et al. 2016; Reddish et al. 2016; Schjoedt et al. 2009; Schaap-Jonker et al. 2013).

\subsection{Absorption}


Absorption, first identified by Tellegen and Atkinson (1974), is a personality trait related to hypnotic susceptibility that also encompasses a broader range of experiences and psychological processes (e.g., imaginative involvement, hyper-focus, full-commitment of attentional resources, situations seeming overly real, and an altered sense of self). Experimental and correlational studies confirm that the brain's attentional system, self-referential processing, and imaginative engagement are core components of trait absorption (for a review, see Roche and McConkey 1990). Importantly, for the current study, these variables may be particularly salient in the realm of mystical experiences, which involve feelings of a narrowing or expanding of conscious attention, facilitating powerful episodes of ego dissolution and union with something larger (Hood 2001). Indeed, Luhrmann (2013) suggests that the psychological capacity for absorption underlies much of what people usually term spiritual or religious experience.

In her psychological and ethnographical study of American Evangelicals in the Vineyard church, Luhrmann (2012) recognized that some congregants reported more intensely vivid and perceptively real divine experiences than others. To better understand this occurrence, Luhrmann, Nusbaum, and Thisted (2010) coded participant interviews for vividness, sensory experience, and focus. After piloting this data against other plausible scales/constructs, they found absorption was the best predictor of individual differences in how the participants reported experiencing God. Moreover, Luhrmann and colleagues found that prayer practice also led to increased intensity of these experiences, suggesting that the frequency of ritual practice, and the length per session, are important variables to consider in future research on experience (Boyer 2013; Luhrmann, Nusbaum and Thisted 2013). Although the religious and spiritual experiences described in Luhrmann et al.'s (2010) study (also see, Luhrmann et al. 2013) contain explicit representational content, such as tactile sensations, ideas or images, which are antithetical to Stace (1960) and 
Hood's (2013) focus on a "common core" to mystical experience, they do contain similar experiences of positive affect and noetic quality. Thus, we expect absorption to function in a similar manner.

\subsection{The current studies}

The present studies were designed as 'exploratory research.' In contrast to confirmatory research, which aims to rigorously test specific hypotheses from an established method or theory, the aim of exploratory research is broader and used to generate new directions and hypotheses for further investigation (for a full discussion see, Charles, Bartlett, Messick, Coleman and Uzdavines 2018; de Groot 1954/2014). This is an important distinction to declare because it affects how our research was designed (for example, favoring a broad research question over very specific hypotheses) and thus how it should be interpreted. Moreover, although this distinction is always implicit in study design, that researchers fail to make this explicit is a major methodological limitation of research in general, and within the psychology of religion in specific (Charles et al. 2018).

In the previous sections, we reviewed evidence that: 1) Mystical experience is central to many religious traditions and human experience more broadly; 2) Mentalizing is a theoretically important foundation of religious belief, however the empirical evidence suggests it may be more relevant for experiences (often deemed religious); 3) Absorption facilitates the cultivation of extraordinarily rich religious or spiritual experiences more generally and is linked specifically to mystical experiences; and 4) Ritual practice can increase the reported intensity and richness of said experiences. Currently, no studies exist testing the interplay between these constructs in general, nor specifically testing their relationship outside of the Christian tradition or examining the role of mentalizing in mystical experiences. Moreover, all previous studies of absorption and mysticism 
have sampled undergraduate university students, and further research is needed drawing from nonstudent samples. The research reported here is an initial attempt to address these issues.

In the present set of exploratory studies, we use hierarchical regression analyses to explore the relationship of mentalizing, absorption, frequency, and duration of ritual practice, on selfreported mystical experiences in a sample of experienced meditators and followers of the Mystic Yogi Sadhguru (study 1), and in an undergraduate psychology student sample (study 2). Moreover, given that previous research has found higher levels of mentalizing related to an increase in religious beliefs and experiences, we explored the possibility that higher levels of mentalizing will moderate the established absorption-mysticism relationship.

\subsection{Study one}

\subsection{Participants}

For study one, we used a unique sample of experienced Isha Foundation meditators. According to its website, the Isha Foundation is a non-profit organization founded in 1992 by the "realized yogi and mystic" Sadhguru Jaggi Vasudev. ${ }^{2}$ Sadhguru is a Shaivite guru from Tamil Nadu India whose centers and temples are spread around the world. While the Isha Foundation sites in India serve more as religious centers, their deployment in the United States focuses on exercises and meditation for healing and self-realization. Like other Hindu gurus who have brought their wisdom west, the foundation and Sadhguru themselves are not without alleged conflict. Both have drawn controversy on topics ranging from the death of Sadhguru's wife, allegations of "brainwashing," and environmental violations. ${ }^{3}$

\footnotetext{
${ }^{2}$ http://adiyogi.org/ and http://www.ishayoga.org/en/about-isha

${ }^{3} \mathrm{https}$ ///isha.sadhguru.org/us/en/blog/article/false-allegations-against-isha-foundation and https://www.patheos.com/blogs/hindu2/2016/08/sadhguru-jaggi-vasudevs-wifes-death-controversy-truth/
} 
As a mystic, Sadhguru seeks to help others achieve their own mystical experiences in the form of realized reality (Coleman, Hood, Holcombe, Swanson, Cao and Giamundo 2016). Many of the programs offered by Isha focus on meditation, yoga, and Ayurvedic teachings related to health and wellness. Like many Eastern traditions which have established themselves in the United States, Isha attempts to appeal to Westerner's interest in ancient Hindu wisdom. Like many Indian gurus before him, Sadhguru suggests his teachings would enrich the west and operates under a similar narrative of sharing ancient wisdom and offering personal insights through self-help (Coleman et al. 2016). Isha is known internationally for its yoga programs and Eastern-orientated contemplative thought, specifically "Inner Engineering," which in their words, "offers a comprehensive process to align your body, mind, emotions, and energy." "2 Moreover, there is some evidence that meditative practices in general can train the capacity for absorption (cf. Luhrmann et al. 2013). The type of practice taught by Sadhguru and the Isha Foundation explicitly encourages the development of one's mystical sense (Coleman et al. 2016). Therefore, our team identified this group as an excellent sample in which to explore absorption, mentalizing, ritual practice, and their association with mysticism.

\subsection{Methods}

The team received approval from our contact at the Isha Foundation (Vinod Sitaraman) to conduct the study. All study materials were submitted to Isha in advance and approved. The manuscript write-up and data analyses are solely the product of co-authors named on this manuscript and have not been reviewed or approved by the Isha Foundation.

Study one was conducted using a Qualtrics online survey, sent from our contact at the Isha Foundation to approximately 1,200 highly devoted meditators. They attended a highly experiential 
ritual event - the consecration of the Adi Yogi Abode - at the Isha Institute of Inner Sciences, located in the foothills of the Appalachian Mountains in McMinnville Tennessee, between September-October 2015. The participants came from across the United States and several other countries, paying upwards of $\$ 800$ to participate in the three-day event. Out of the roughly 1200 event participants, 412 completed the survey and 269 (146 female; $M$ age $=43.03$ years, $S D=$ 10.82) had full data for the variables included in the regression model. Given the exploratory nature of the study, an a priori power analysis was not conducted. However, a sensitivity power analysis (G*Power; Faul et al. 2009) conducted after the participants were recruited shows that 269 participants would make a linear regression model with seven predictors sensitive enough to detect a 0.03 increase in $\mathrm{R}^{2}(\alpha=.05$, power $=.80)$.

Self-reported mystical experiences were measured using Hood's (1975, 2001) M-Scale (e.g. "I have had an experience which was both timeless and spaceless."; Cronbach's $\alpha=0.95$, $95 \% \mathrm{CI}=[0.94,0.96])$. Individual differences in ToM ability (mentalizing) was measured with the Empathizing Quotient short form (Wakabayashi et al. 2006; which measures self and other directed mentalization, and has been used in several studies examining belief in God: e.g., Greenway 2018; Norenzayan et al. 2012; Wlodarski and Pearce 2016) (e.g. "I am good at predicting how someone will feel.”; $\alpha=0.88,95 \% \mathrm{CI}=[0.86,0.9])$. The Modified Tellegen Absorption Scale (Jamieson 2005) was utilized to measure Trait Absorption (e.g. "The crackle and flames of a wood fire stimulate my imagination."; $\alpha=0.94,95 \% \mathrm{CI}=[0.93,0.95]) .{ }^{4}$

\subsection{Results}

\footnotetext{
${ }^{4}$ See the original articles for further information on scoring.
} 
A linear regression model was created with the interaction term of absorption and mentalizing used to predict mystical experiences. Age, gender, weekly meditative practice, and time per meditative session were also entered into the model as predictors. Statistical analyses were conducted in R (R Core Team 2017) ${ }^{5}$. The data and analysis scripts are available on the Open Science Framework (https://osf.io/vb36p/?view_only=fd0bd39996dd428cad25cba24acf3a59).

The final regression model was determined by entering the variables in four stages in a hierarchical process. The model with the best fit and smallest Akaike Information Criterion (AIC) was retained (Baguley 2012). The AIC indicates the quality of a regression model relative to another model, but penalizes more complicated models. The first model consisted of age and gender and explained a small but significant amount of variance in mystical experience $(F(2,266)$ $\left.=9.93, p<.001, R^{2}=0.07, \mathrm{AIC}=2541.45\right)$. A process of model comparison was then performed which compared the increase in $R^{2}$ from the previous model. The addition of weekly meditative practice and time per session significantly increased the amount of variance explained by the model $\left(F(2,264)=11.51, p<.001, R^{2}=0.12, \mathrm{AIC}=2529.36\right)$. Absorption and mentalizing were entered as individual predictors in the third model which again significantly increased the amount of explained variance $\left(F(2,262)=53.57, p<.001, R^{2}=0.38, \mathrm{AIC}=2442.35\right)$. Finally, the interaction between absorption and mentalizing was entered into the fourth model $(F(1,261)=5.15, p=.024$, $R^{2}=0.39$, AIC $\left.=2439.1\right)$. The fourth model was retained as our final model as it explained the most variance and had the smallest AIC value, suggesting the best model relative to the others (Baguley 2012). Descriptive statistics for each variable included in the final model in studies one and two are reported in Table 1, and the correlation matrix for each variable is reported in Table

\footnotetext{
${ }^{5}$ The following R packages were used cowplot (Wilke 2017); jtools (Long 2018); MBESS (Kelley 2017); psych (Revelle 2017); stargazer (Hlavac 2018); sjPlot (Lüdecke 2018); Tidyverse (Wickham 2017).
} 
2. An additional correlation matrix with $95 \%$ confidence intervals can be found online in the OSF project.

Table 1. Descriptive statistics

\begin{tabular}{lcccccc}
\hline & \multicolumn{3}{c}{ Study one $(N=269)$} & \multicolumn{3}{c}{ Study two $(N=123)$} \\
\hline & $M$ & $S D$ & Range & $M$ & $S D$ & Range \\
\hline Mystical & 119.35 & 27.88 & $40-160$ & 114.37 & 17.54 & $54-149$ \\
experiences & 92.74 & 22.98 & $43-154$ & 95.93 & 21.15 & $43-152$ \\
Absorption & 24.89 & 8.52 & $4-43$ & 25.77 & 7.95 & $5-43$ \\
Mentalizing & 43.03 & 10.82 & $22-79$ & 20.69 & 4.52 & $18-44$ \\
Age & 4.44 & 0.97 & $1-6$ & 7.96 & 10.12 & $0-49$ \\
Weekly meditation & 4.38 & 1.34 & $1-5$ & 9.85 & 15.57 & $0-60$ \\
practice* & & &
\end{tabular}

Note: ${ }^{*}$ In study two this was called weekly religious/spiritual practice.

Table 2. Correlation matrix for variables included in study one

\begin{tabular}{lcccccc}
\hline & 1. & 2. & 3. & 4. & 5. & 6. \\
\hline 1.Age & 1 & 0.08 & -0.02 & 0.09 & $0.17^{*}$ & 0.06 \\
2.Weekly meditation practice & & 1 & $0.48^{*}$ & 0.09 & 0.01 & $0.23^{*}$ \\
3.Minutes per session & & & 1 & 0.09 & -0.02 & $0.20^{*}$ \\
4.Absorption & & & & 1 & $0.38^{*}$ & $0.55^{*}$ \\
5.Mentalizing & & & & & 1 & $0.36^{*}$ \\
6.Mystical experiences & & & & & 1 \\
\hline
\end{tabular}

Note: ${ }^{*} p<.05$ corrected for multiple comparisons using Holm correction (Holm 1979).

The final model explained $38.7 \%\left(\operatorname{adj} . R^{2}=0.37\right)$ of the variance in mysticism, and the full results are reported in Table 3. Crucially, there was a significant interaction between absorption and mentalizing. To gain a better understanding of the interaction, a simple slopes analysis was performed to explore the effect of different mentalizing values on the slope of absorption. This suggests there is a positive relationship between mystical experiences and absorption. The simple slopes analysis showed that this relationship is moderated by mentalizing, with the relationship 
between mystical experiences and absorption being stronger with lower values of mentalizing.

This moderating effect is shown in Figure 1.

Table 3. Linear model of predictors of mystical experiences in study one

\begin{tabular}{|l|c|c|c|c|c|}
\hline & $b$ & $95 \% C I$ & $S E$ & $t$ & $p$ \\
\hline Constant & 117.48 & $113.22,121.74$ & 2.17 & 54.28 & $p<.001$ \\
\hline Absorption & 0.55 & $0.42,0.68$ & 0.07 & 8.26 & $p<.001$ \\
\hline Mentalizing & 0.56 & $0.22,0.91$ & 0.18 & 3.22 & $p<.001$ \\
\hline Absorption x mentalizing & -0.02 & $-0.03,-0.002 *$ & 0.01 & -2.27 & $p=.02$ \\
\hline Age & -0.13 & $-0.39,0.13$ & 0.13 & -0.97 & $p=.33$ \\
\hline Gender & 5.70 & $-0.09,11.48$ & 2.94 & 1.94 & $p=.05$ \\
\hline Weekly meditation practice & 3.96 & $0.82,7.10$ & 1.59 & 2.48 & $p=.01$ \\
\hline Minutes per session & 2.24 & $-0.06,4.54$ & 1.17 & 1.92 & $p=.06$ \\
\hline
\end{tabular}

$F(7,261)=23.56, p<.01, R^{2}=0.39,95 \% \mathrm{CI}=[.28, .46]$, adj. $R^{2}=0.37, \mathrm{AIC}=2439.10$.

Note: all predictors were centered in the model. *This value is intentionally rounded to three decimal places to prevent it being misleadingly reported as -0.00 .

The slope decreases from when mentalizing is one standard deviation below the mean

$(0.69, \mathrm{SE}=0.1,95 \% \mathrm{CI}=[0.5,0.89], p<.001)$, to the mean $(0.55, \mathrm{SE}=0.07,95 \% \mathrm{CI}=[0.42$,

$0.68], p<.001)$, and to one standard deviation above the mean $(0.41, \mathrm{SE}=0.08,95 \% \mathrm{CI}=[0.25$,

0.57], $p<.001)$. This shows that when mentalizing is lower, there is a stronger relationship between absorption and mystical experiences. 


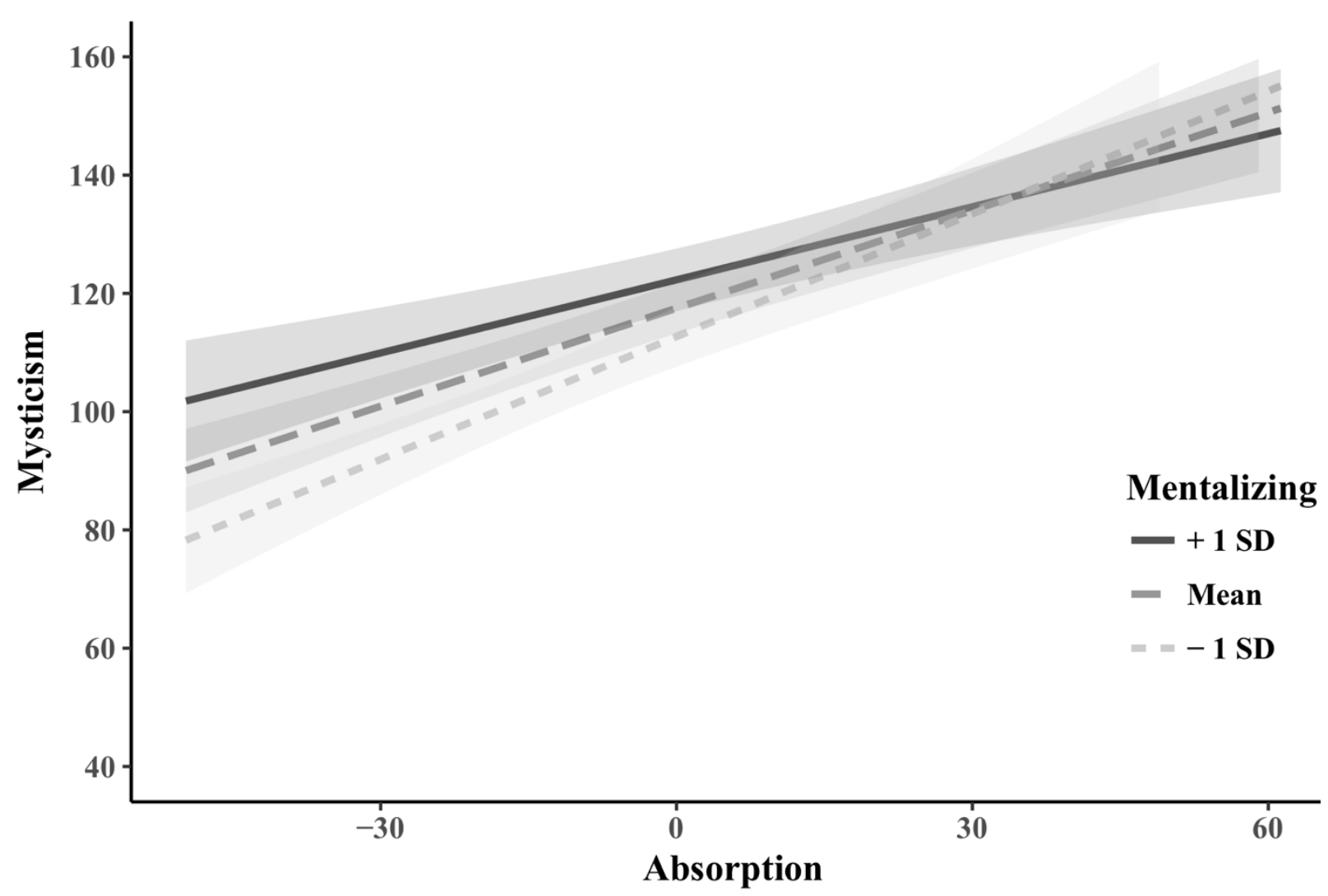

Figure 1.

Moderating effect of mentalizing on the relationship between mystical experiences and absorption. The relationship between mystical experiences and absorption is stronger for lower values of the moderator mentalizing. The shaded bands around the regression lines show the $95 \%$ confidence interval. Note that the predictors are centered, and the y-axis does not contain 0 as the minimum value for mysticism is 40 .

\subsection{Study two}

\subsection{Participants}

To investigate if the results from study one generalized to a different sample, the same modelling procedure was run on a sample of 144 undergraduate psychology students from a predominantly Christian context at a medium sized southern university. The final sample consisted of 123 participants $(89$ female; $M$ age $=20.69$ years, $S D=4.52)$. An a priori power analysis was not conducted for this study. A sensitivity power analysis conducted after the participants were recruited showed that 123 participants would make a model with seven predictors sensitive enough 
to detect a 0.06 increase in $R^{2}(\alpha=.05$, power $=.80)$. The data were collected in two stages. In order to control for an increase in type I errors from performing interim analyses, a corrected alpha value of .029 was used for one interim and one final analysis (Lakens 2014).

\subsection{Methods}

The survey was identical to study one with one exception. The weekly practice question was changed from "meditative practice" to "religious and/or spiritual practice" to be more relatable to the sample: M-Scale (Cronbach's $\alpha=0.88,95 \% \mathrm{CI}=[0.85,0.91])$; mentalizing $(\alpha=0.88,95 \% \mathrm{CI}$ $=[0.85,0.91])$; and Trait Absorption $(\alpha=0.93,95 \% \mathrm{CI}=[0.91,0.95])$. See Table 1 for each scale mean, standard deviation, and range.

\subsection{Results}

Similar to study one, the final model was determined in a hierarchical process. The first model consisted of age and gender which explained a small, non-significant (based on our corrected alpha value) amount of variance in mystical experiences $\left(F(2,120)=3.35, p=.039, R^{2}=0.05\right.$, AIC $=$ 1053.99). The addition of weekly religious/spiritual practice and time per session increased the amount of variance explained in the second model $\left(F(2,118)=4.86, p=.009, R^{2}=0.1\right.$, AIC $=$ 1051.11). The addition of absorption and mentalizing in model three further increased the amount of variance explained by the model $\left(F(2,116)=26.70, p<.001, R^{2}=0.39\right.$, AIC $\left.=1008.35\right)$. However, in contrast to study one, the addition of the interaction between absorption and mentalizing did not improve the model $\left(F(1,115)=0.46, p=.499, R^{2}=0.39\right.$, AIC $\left.=1009.86\right)$. Model three was retained as the best model, without the interaction between absorption and mentalizing. The correlation matrix for each variable included in the final model is shown in Table 
4. An additional correlation matrix with $95 \%$ confidence intervals can be found online in the OSF project. The final model is shown in Table 5.

Table 4. Correlation matrix for variables included in study two

\begin{tabular}{lcccccc}
\hline & 1. & 2. & 3. & 4. & 5. & 6. \\
\hline 1. Age & 1 & 0.02 & 0.09 & 0.08 & -0.07 & 0.10 \\
2. Weekly religious/spiritual practice & & 1 & 0.16 & -0.02 & 0.01 & 0.19 \\
3. Time per session & & & 1 & 0.14 & 0.01 & 0.15 \\
4. Absorption & & & & 1 & $0.27^{*}$ & $0.54^{*}$ \\
5. Mentalizing & & & & & 1 & 0.25 \\
6. Mystical experiences & & & & & 1 \\
\hline
\end{tabular}

Note: ${ }^{*} p<.05$ corrected for multiple comparisons using Holm correction (Holm 1979).

The model explained $38.76 \%\left(95 \% \mathrm{CI}=[0.22,0.5]\right.$, adj. $\left.R^{2}=0.36\right)$ of the variance in mystical experiences. However, in comparison to study one, there was no significant interaction term, and the only significant predictors were absorption, gender, and weekly religious/spiritual practice.

Table 5. Linear model of predictors of mystical experiences in study two

\begin{tabular}{lccccc}
\hline & $b$ & $95 \% C I$ & $S E$ & $t$ & $p$ \\
\hline Constant & 108.64 & $103.81,113.46$ & 2.44 & 44.56 & $p<.001$ \\
Absorption & 0.42 & $0.29,0.54$ & 0.06 & 6.57 & $p<.001$ \\
Mentalizing & 0.22 & $-0.11,0.55$ & 0.17 & 1.30 & $p=.20$ \\
Age & 0.32 & $-0.25,0.88$ & 0.29 & 1.10 & $p=.27$ \\
Gender & 7.93 & $2.24,13.62$ & 2.88 & 2.76 & $p=.01$ \\
Weekly religious/spiritual practice & 0.35 & $0.10,0.60$ & 0.13 & 2.73 & $p=.01$ \\
Time per session & 0.05 & $-0.12,0.21$ & 0.08 & 0.55 & $p=.58$ \\
\hline
\end{tabular}

$F(6,116)=12.24, p<.01, R^{2}=.39,95 \% \mathrm{CI}=[.22, .50]$, adj. $R^{2}=.36$

Note: all predictors were centered in the model.

\subsection{Discussion}

Across two studies, the relationship between mentalizing, trait absorption, frequency and duration of spiritual or meditative practice, and self-reported mystical experiences was investigated. The results support previous research indicating the importance of absorption for 
mystical experiences (Granqvist et al. 2005; Granqvist et al. 2012; Spanos and Moretti 1988). Moreover, study one provides the first evidence for this relationship in a non-student sample, as well as demonstrating a potentially surprising finding-lower mentalizing ability enhanced the relationship between absorption and self-reported mystical experiences. Study two replicated the absorption-mysticism link, however mentalizing was no longer a significant predictor or moderator. Below, we examine the implications of these results in greater detail.

Practice and mysticism: Following James' (1902) distinctly Protestant approach, most scholarship has focused on purported spontaneous bouts of mystical experience (Luhrmann 2013). This has obscured the significant contribution of ritual practices and training in developing expertise entering into mystical states, which can serve to validate the reality of things unseen from the experiencer's perspective (Boyer 2013; Lurhmann 2012). Neither study identified a link between the length of practice and mystical experiences. However, linear regression analyses indicated that frequency of practice (times per week) was a predictor of self-reported mystical experiences in both samples, which is potentially interesting given their different characteristics.

For example, the meditators in study one received specific training and encouragement from Sadhguru and the Isha Foundation to engage regularly in meditative practices with the explicit aim of cultivating their "mystical sense." In comparison, the student sample hailed from a Christian context. Although we cannot be certain of the type of practice, aim, and training of the students, it is likely that they attended church, prayed, but did not specifically focus on cultivating a mystical sense, and lacked explicit training (this was partly reflected in the few open responses for weekly practice). Therefore, one interpretation is that, at least in the current two samples, differences in training, type, and aim of practice may be less important for the cultivation of mystical experiences as measured by the M-Scale. However, this seems unlikely given that the 
student sample reported twice as much ritual practice yet had M-Scale means several points lower than the Isha meditators. In other words, the Isha meditators seem to be getting more experience, but with less effort, suggesting skill does play a role. Regardless, in both studies, hierarchical regression analyses indicated that frequency of ritual practice contributed minimally to the explained variance in mysticism scores. This suggests that when practice influences the occurrence of self-reported mystical experiences, it does so weakly.

Absorption and mysticism: Tellegen and Atkinson (1974) suggest that people high in absorption may be fond of mystical experiences. Across two studies, we found support for the link between absorption and self-reported mystical experiences. Further, we provided the first evidence for this relationship in a non-student sample (Isha meditators). These results add to a small but growing literature suggesting individual differences in trait absorption contribute to variation in the self-report of mystical experiences. Moreover, compared to the other variables we analyzed, the regression model indicates that absorption contributes most strongly to the explained variance in mysticism scores. Experimental studies suggest individuals high in absorption may be predisposed to perceived mystical, unusual, and hallucinatory experiences (Granqvist et al. 2005; Luhrmann 2013; van Elk 2015). The full engagement of attention takes on an absorbing-like quality which includes experiencing a temporary loss of self or union with the attending thought/object and therefore contributes to the experiential dimensions of self-reported mystical experiences.

Mentalizing and mysticism: Self-report measures of mentalizing have demonstrated mixed importance for belief in supernatural agents. However, they have been more consistently related to reported experiences (see section 1.3). In study one, we provided the first evidence that mentalizing is a predictor of self-reported mystical experiences. In contrast, mentalizing was not a 
significant predictor of mysticism in study two. Further research is required to better understand what specific aspects of religious or mystical experience are related to mentalizing ability and in what samples might we expect this relationship.

Absorption-mentalizing-mysticism: In contrast to our expectation that higher levels of mentalizing might moderate the link between absorption and mysticism, the opposite occurred. In study one, there was a stronger relationship between absorption and mysticism for individuals with lower levels of mentalizing. Due to the theoretical importance of mentalizing for experiences deemed religious and/or mystical (Cristofori et al 2016; van Elk and Aleman 2017), this is a potentially interesting interaction. It suggests that, although experiences of the divine are mediated by mentalizing processes (Kapogiannis et al. 2009; Reddish et al. 2016; Schaap-Jonker et al. 2013), trait absorption may facilitate these experiences when mentalizing ability is lower than normal. In part, our current finding was foreshadowed by Cristofori et al. (2016), who intimated the possibility that attentional processes (such as those captured by absorption) might be able to facilitate mystical experiences without substantial input from mentalizing processes. However, in study two we were unable to replicate the interaction between absorption and mentalizing. Considering the unique characteristics of the Isha meditators when compared to the student sample used in study two that we discussed previously, it is difficult to interpret this null finding because the samples vary considerably. Such is the plight of this type of exploratory research, and future studies will be needed to better understand the partial findings reported here.

\section{Conclusion}

This exploratory research sought to broaden the relevance of ToM for the cognitive science of religion by exploring the role of mentalizing and absorption in the self-report of mystical 
experience. Our data supports recent suggestions that a shift toward focusing on experience and affect may be a fruitful yet understudied area of investigation (Coleman, Jong and van Mulukom 2018; Luhrmann 2013; Tan 2018). In addition to replicating the results of the present study, it would be interesting for future research to study the relationship between absorption, mentalizing, and mysticism in samples of neurotypicals with low mentalizing abilities and special populations with atypical mentalizing traits (e.g., autism spectrum). Although the belief-orientated aspects of religion may be difficult for low mentalizers, mentalizing abilities may actually hinder other psychological processes, such as absorption, that can enhance the more experiential dimensions of mystical and other special experiences. Our understanding of the cognitive underpinnings of mystical experience is in its infancy and we hope the findings reported here will be useful for further theoretical reflection and empirical investigation.

\section{Acknowledgments}

This research was conducted while Coleman and Swanson were at The University of Tennessee at Chattanooga. Coleman thanks the Isha Foundation for travel funding to present an earlier draft of this paper at the 2016 International Association for Cognitive Science of Religion conference. The co-authors are grateful to the Isha Foundation for their cooperation while conducting study 1 , specifically our contacts, Vinod and Jyoti.

We are grateful for the feedback of three anonymous reviewers on an earlier version of this article. 


\section{References}

Andersen, Marc, K.L. Nielbo, U. Schjoedt, and J. Sørensen. 2014. "Mystical Experience in The Lab". Method \& Theory in The Study of Religion 26 (3): 217-245. doi:10.1163/1570068212341323.

Baguley, Thomas. 2012. Serious Stats: A Guide to Advanced Statistics for the Behavioural Sciences. Basingstoke: Palgrave MacMillan.

Barrett, Justin L. 2004. Why Should Anyone Believe in God?. Walnut Creek, CA: AltaMira Press.

Beauregard, Mario, and Vincent Paquette. 2006. "Neural Correlates of a Mystical Experience in Carmelite Nuns". Neuroscience Letters 405 (3): 186-190. doi:10.1016/j.neulet.2006.06.060.

Boyer, Pascal, and Brian Bergstrom. 2008. "Evolutionary Perspectives on Religion". Annual Review of Anthropology 37 (1): 111-130. doi:10.1146/annurev.anthro.37.081407.085201.

Boyer, Pascal. 1992. "Explaining Religious Ideas: Elements of A Cognitive Approach". Numen 39 (1): 27. doi:10.2307/3270074.

Boyer, Pascal. 2013. "Why "Belief” Is Hard Work". HAU: Journal of Ethnographic Theory 3 (3): 349-357. doi:10.14318/hau3.3.015.

Brezis, Rachel. 2012. "Autism as a Case for Neuroanthropology: Delineating the Role of Theory of Mind in Religious Development". In the Encultured Brain, 291-314. Boston: MIT Press.

Caldwell-Harris, Catherine, Caitlin Fox Murphy, Tessa Velazquez, and Patrick McNamara. 2011. "Religious Belief Systems of Persons with High Functioning Autism". In , 3362-3366. Proceedings of the Annual Meeting of the Cognitive Science Society.

Coleman III, Thomas J., Jonathan Jong, and Valerie van Mulukom. 2018. "Introduction to The Special Issue: What Are Religious Beliefs?". Contemporary Pragmatism 15 (3): 279-283. doi:10.1163/18758185-01503001.

Coleman III, Thomas J., Ralph W. Hood Jr., Jenny M. Holcombe, Sally B. Swanson, Juan Cao, and Derek Giamundo. 2016. "When Shiva Talks Back: Absorption, Mentalizing, and Mysticism". Presentation, 6 th biennial meeting of the International Association for the Cognitive Science of Religion, , 2016.

Coleman III, Thomas. 2016. "The Social Brain in Human and Religious Evolution: Elucidating the Role of Theory of Mind In (Non)Religious Belief". Master's thesis, The University of Tennessee at Chattanooga.

Coleman, Thomas, Ann Swhajor - Biesemann, Derek Giamundo, Christopher Vance, Ralph W. Hood Jr., and Christopher F. Silver. 2016. "“Experimenting with Ideologies..." - A "More 
Spiritual Than Religious" Zen Buddhist". In Semantics and Psychology of Spirituality, 339353. Dordrecht: Springer.

Charles, Sarah J., James E. Bartlett, Kyle J. Messick, Thomas J. Coleman III, and Alex Uzdavines. 2018. "Researcher Degrees of Freedom in the Psychology of Religion." PsyArXiv. December 22. doi:10.31234/osf.io/tx5gd

Cristofori, Irene, Joseph Bulbulia, John H. Shaver, Marc Wilson, Frank Krueger, and Jordan Grafman. 2016. "Neural Correlates of Mystical Experience". Neuropsychologia 80: 212-220. doi:10.1016/j.neuropsychologia.2015.11.021.

Dahl, Cortland J., Antoine Lutz, and Richard J. Davidson. 2015. "Reconstructing and Deconstructing the Self: Cognitive Mechanisms in Meditation Practice". Trends in Cognitive Sciences 19 (9): 515-523. doi:10.1016/j.tics.2015.07.001.

Edman, Laird, Chris Sietstra, Molly Townsend, Corey Kundert, Hope DeRuyter, Rebekah Muilenberg, Virgina Kjer, and Riley Harder. 2018. "Mentalizing, Personal Prayer, the Presence of God, and Evil," Northwestern Review: 3 (1): Available at https://nwcommons.nwciowa.edu/northwesternreview/vol3/iss1/7

Faul, Franz, Edgar Erdfelder, Axel Buchner, and Albert-Georg Lang. 2009. "Statistical Power Analyses Using G*Power 3.1: Tests for Correlation and Regression Analyses". Behavior Research Methods 41 (4): 1149-1160. doi:10.3758/brm.41.4.1149.

Ferguson, Michael A., Jared A. Nielsen, Jace B. King, Li Dai, Danielle M. Giangrasso, Rachel Holman, Julie R. Korenberg, and Jeffrey S. Anderson. 2018. "Reward, Salience, And Attentional Networks Are Activated by Religious Experience in Devout Mormons". Social Neuroscience 13 (1): 104-116. doi:10.1080/17470919.2016.1257437.

Gervais, Will M. 2013. "Perceiving Minds and Gods". Perspectives on Psychological Science 8 (4): 380-394. doi:10.1177/1745691613489836.

Granqvist, Pehr, Berit Hagekull, and Tord Ivarsson. 2012. "Disorganized Attachment Promotes Mystical Experiences Via A Propensity for Alterations in Consciousness (Absorption)". International Journal for The Psychology of Religion 22 (3): 180-197. doi:10.1080/10508619.2012.670012.

Granqvist, Pehr, Mats Fredrikson, Patrik Unge, Andrea Hagenfeldt, Sven Valind, Dan Larhammar, and Marcus Larsson. 2005. "Sensed Presence and Mystical Experiences Are Predicted by Suggestibility, Not by The Application of Transcranial Weak Complex Magnetic Fields". Neuroscience Letters 379 (1): 1-6. doi:10.1016/j.neulet.2004.10.057.

Greenway, Tyler S. 2018. "Mentalizing Domains and Belief in God". Journal for The Cognitive Science of Religion 4 (1): 91-110. doi:10.1558/jcsr.31063. 
Harris, Sam, Jonas T. Kaplan, Ashley Curiel, Susan Y. Bookheimer, Marco Iacoboni, and Mark S. Cohen. 2009. "The Neural Correlates of Religious and Nonreligious Belief". Plos ONE 4 (10): e7272. doi:10.1371/journal.pone.0007272.

Hlavac, Marek. 2018. stargazer: Well-Formatted Regression and Summary Statistics Tables. R package version 5.2.1. https://CRAN.R-project.org/package=stargazer

Hood, Ralph W, Peter C Hill, and Bernard Spilka. 2018. The Psychology of Religion: An Empirical Approach. 5th ed. New York: Guilford Press.

Hood, Ralph W. 1977. "Eliciting Mystical States of Consciousness with Semistructured Nature Experiences". Journal for The Scientific Study of Religion 16 (2): 155. doi:10.2307/1385746.

Hood, Ralph W. 2013. "Theory and Methods in The Psychological Study of Mysticism". International Journal for The Psychology of Religion 23 (4): 294-306. doi:10.1080/10508619.2013.795803.

Hood, Ralph W. 2001. Dimensions of Mystical Experiences. Amsterdam: Rodopi.

Hood, Ralph W. 1995. Handbook of Religious Experience. Birmingham, Ala.: Religious Education Press.

Jack, Anthony Ian, Jared Parker Friedman, Richard Eleftherios Boyatzis, and Scott Nolan Taylor. 2016. "Why Do You Believe in God? Relationships Between Religious Belief, Analytic Thinking, Mentalizing And Moral Concern". PLOS ONE 11 (3): e0149989. doi:10.1371/journal.pone.0149989.

James, William. 1902. Th E Varieties of Religious Experience. Cambridge, MA: Harvard University Press.

Jamieson, Graham A. 2005. "The Modified Tellegen Absorption Scale: A Clearer Window on The Structure and Meaning of Absorption". Australian Journal of Clinical \& Experimental Hypnosis 33 (2): 119-139.

Kapogiannis, Dimitrios, Aron K. Barbey, Michael Su, Giovanna Zamboni, Frank Krueger, and Jordan Grafman. 2009. "Cognitive and Neural Foundations of Religious Belief". Proceedings of The National Academy of Sciences 106 (12): 4876-4881. doi:10.1073/pnas.0811717106.

Katz, Steven T. 1978. Mysticism and Philosophical Analysis. New York: Oxford University Press.

Kelley, Ken. 2017. MBESS: The MBESS R Package. R package version 4.3.0. https://CRAN.Rproject.org/package $=$ MBESS 
Klein, Constantin, Christopher F. Silver, Heinz Streib, Ralph W. Hood Jr., and Thomas J. Coleman. 2016. "Mysticism (The M-Scale) And "Spirituality". In The Semantics and Psychology of Spirituality, 1st ed., 165-187. Dordrecht: Springer.

Laidlaw, Tannis M., Prabudha Dwivedi, Akira Naito, and John H. Gruzelier. 2005. "Low SelfDirectedness (TCI), Mood, Schizotypy And Hypnotic Susceptibility". Personality and Individual Differences 39 (2): 469-480. doi:10.1016/j.paid.2005.01.025.

Lakens, Daniël. 2014. "Performing High-Powered Studies Efficiently with Sequential Analyses". European Journal of Social Psychology 44 (7): 701-710. doi:10.1002/ejsp.2023.

Levine, Jeffrey, Ian E. Wickramasekera, and Caryle Hirshberg. 1998. "Is Religiousness A Correlate of Absorption? Implications for Psychophysiology, Coping, and Morbidity". Alternative Therapies in Health and Medicine 4 (6): 72-76.

Lewis-Williams, David. 2010. Conceiving God: The Cognitive Origin and Evolution of Religion. London: Thames \& Hudson.

Lindeman, Marjaana, and Jari Lipsanen. 2017. "Mentalizing: Seeking the Underlying Dimensions". International Journal of Psychological Studies 9 (1): 10. doi:10.5539/ijps.v9n1p10.

Long, Jacob. 2018. jtools: Analysis and Presentation of Social Scientific Data. R package version 1.0.0, URL: https://cran.r-project.org/package=jtools.

Lüdecke Daniel. 2018. sjPlot: Data Visualization for Statistics in Social Science. R package version 2.4.1, URL: https://CRAN.R-project.org/package=sjPlot.

Luhrmann, Tanya M. 2012. When God Talks Back. New York: Vintage Books.

Luhrmann, Tanya M., Howard Nusbaum, and Ronald Thisted. 2010. "The Absorption Hypothesis: Learning to Hear God in Evangelical Christianity". American Anthropologist 112 (1): 66-78. doi:10.1111/j.1548-1433.2009.01197.x.

Luhrmann, Tanya M., Howard Nusbaum, and Ronald Thisted. 2013. "“Lord, Teach Us to Pray": Prayer Practice Affects Cognitive Processing". Journal of Cognition and Culture 13 (1-2): 159-177. doi:10.1163/15685373-12342090.

Luhrmann, Tanya M. 2013. "Building on William James: The Role of Learning in Religious Experience". In Mental Culture: Classical Social Theory and The Cognitive Science of Religion, 145-163. Acumen Publishing.

Maij, David. L. R., Frenk van Harreveld, Will Gervais, Yann Schrag, Christine Mohr, and Michiel van Elk. 2017. "Mentalizing Skills Do Not Differentiate Believers from Non-Believers, But Credibility Enhancing Displays Do". PLOS ONE $12 \quad$ (8): e0182764. doi:10.1371/journal.pone.0182764. 
McCauley, Robert N. 2011. Why Religion Is Natural and Science Is Not. Oxford University Press.

McNamara, Amelia, de la Rubia, Eduardo, Zhu, Hao, Ellis, Shannon, and Quinn, Michael. 2018. skimr: Compact and Flexible Summaries of Data. R package version 1.0.2. URL: https://github.com/ropenscilabs/skimr

Mitchell, Jason P. 2009. "Inferences About Mental States". Philosophical Transactions of the Royal Society B: Biological Sciences 364 (1521): 1309-1316. doi:10.1098/rstb.2008.0318.

Norenzayan, Ara, Will M. Gervais, and Kali H. Trzesniewski. 2012. "Mentalizing Deficits Constrain Belief in a Personal God". Plos ONE 7 (5): $\mathrm{e} 36880$. doi:10.1371/journal.pone.0036880.

Partridge, Christopher H, and Theodore P. C Gabriel. 2003. Mysticisms East and West. Carlisle: Paternoster.

R Core Team. 2017. R: A language and environment for statistical computing. R Foundation for Statistical Computing, Vienna, Austria. URL https://www.R-project.org/.

Reddish, Paul, Penny Tok, and Radek Kundt. 2016. "Religious Cognition and Behaviour In Autism: The Role of Mentalizing". The International Journal for The Psychology of Religion 26 (2): 95-112. doi:10.1080/10508619.2014.1003518.

Revelle, W. 2017. psych: Procedures for Personality and Psychological Research, Northwestern University, Evanston, Illinois, USA, https://CRAN.R-project.org/package=psych Version $=$ 1.7.5.

Roche, Suzanne M., and Kevin M. McConkey. 1990. "Absorption: Nature, Assessment, and Correlates.". Journal of Personality and Social Psychology 59 (1): 91-101. doi:10.1037/00223514.59.1.91.

Rosenkranz, Patrick, and Bruce G. Charlton. 2013. "Individual Differences in Existential Orientation: Empathizing and Systemizing Explain The Sex Difference in Religious Orientation and Science Acceptance". Archive for The Psychology of Religion 35 (1): 119-146. doi:10.1163/15736121-12341255.

Rossano, Matt J. 2006. "The Religious Mind and The Evolution of Religion.". Review of General Psychology 10 (4): 346-364. doi:10.1037/1089-2680.10.4.346.

Schaap-Jonker, Hanneke, Bram Sizoo, Jannine van Schothorst-van Roekel, and Jozef Corveleyn. 2013. "Autism Spectrum Disorders and The Image of God as a Core Aspect of Religiousness". International Journal for The Psychology of Religion 23 (2): 145-160. doi:10.1080/10508619.2012.688005.

Schjoedt, Uffe, Hans Stødkilde-Jørgensen, Armin W. Geertz, Torben E. Lund, and Andreas Roepstorff. 2011. "The Power of Charisma-Perceived Charisma Inhibits the Frontal 
Executive Network of Believers in Intercessory Prayer". Social Cognitive and Affective Neuroscience 6 (1): 119-127. doi:10.1093/scan/nsq023.

Schjoedt, Uffe, Hans Stødkilde-Jørgensen, Armin W. Geertz, and Andreas Roepstorff. 2009. "Highly Religious Participants Recruit Areas of Social Cognition in Personal Prayer". Social Cognitive and Affective Neuroscience 4 (2): 199-207. doi:10.1093/scan/nsn050.

Spanos, Nicholas P., and Patricia Moretti. 1988. "Correlates of Mystical and Diabolical Experiences in a Sample of Female University Students". Journal for The Scientific Study of Religion 27 (1): 105. doi:10.2307/1387405.

Stace, W.T. 1960. Mysticism and Philosophy. Philadelphia: Lippincott.

Tan, Tobias. 2018. "William James And Embodied Religious Belief". Contemporary Pragmatism 15 (3): 366-386. doi:10.1163/18758185-01503006.

Taves, Ann. 1999. Fits, Trances, \& Visions. Princeton, N.J.: Princeton University Press.

Tellegen, Auke, and Gilbert Atkinson. 1974. "Openness to Absorbing and Self-Altering Experiences ("Absorption"), A Trait Related to Hypnotic Susceptibility.". Journal of Abnormal Psychology 83 (3): 268-277. doi:10.1037/h0036681.

van Elk, Michiel, and André Aleman. 2017. "Brain Mechanisms in Religion and Spirituality: An Integrative Predictive Processing Framework". Neuroscience \& Biobehavioral Reviews 73: 359-378. doi:10.1016/j.neubiorev.2016.12.031.

van Elk, Michiel. 2015. "An EEG Study on The Effects of Induced Spiritual Experiences on Somatosensory Processing and Sensory Suppression". Journal for The Cognitive Science of Religion 2 (2): 121-157. doi:10.1558/jcsr.v2i2.24573.

Van Leeuwen, Neil, and Michiel van Elk. 2018. "Seeking the Supernatural: The Interactive Religious Experience Model". Religion, Brain \& Behavior, 1-31. doi:10.1080/2153599x.2018.1453529.

Visuri, Ingela. 2012. "Could Everyone Talk to God? A Case Study on Asperger's Syndrome, Religion, and Spirituality". Journal of Religion, Disability \& Health 16 (4): 352-378. doi:10.1080/15228967.2012.731888.

Wakabayashi, Akio, Simon Baron-Cohen, Sally Wheelwright, Nigel Goldenfeld, Joe Delaney, Debra Fine, Richard Smith, and Leonora Weil. 2006. "Development of Short Forms of the Empathy Quotient (EQ-Short) and the Systemizing Quotient (SQ-Short)". Personality and Individual Differences 41 (5): 929-940. doi:10.1016/j.paid.2006.03.017.

Wickham, Hadley. 2017. tidyverse: Easily Install and Load the 'Tidyverse'. R package version 1.2.1. https://CRAN.R-project.org/package=tidyverse 
Wickramasekera, Ian E. 2015. "Mysteries of Hypnosis and The Self Are Revealed by The

Psychology and Neuroscience of Empathy". American Journal of Clinical Hypnosis 57 (3):

330-348. doi:10.1080/00029157.2014.978495.

Wiebe, Donald. 2013. "The Significance of The Natural Experience of a "Non-Natural" World to The Question of The Origin of Religion". In Origins of Religion, Cognition and Culture, 140159. Acumen Publishing.

Willard, Aiyana K., and Ara Norenzayan. 2017. "'Spiritual but Not Religious": Cognition, Schizotypy, and Conversion in Alternative Beliefs". Cognition 165: 137-146. doi:10.1016/j.cognition.2017.05.018.

Wilke, Claus (2017). cowplot: Streamlined Plot Theme and Plot Annotations for 'ggplot2'. R package version 0.8.0. https://CRAN.R-project.org/package=cowplot

Wlodarski, Rafael, and Eiluned Pearce. 2016. "The God Allusion". Human Nature 27 (2): 160172. doi:10.1007/s12110-016-9256-9. 